An

\title{
Investigation of the Physical Properties of PLZT Ferroelectric Ceramics - Effect of the Lanthanum Content
}

\author{
J. D. S. Guerra, A. C. Silva, R. Mcintosh, Mohammad M. Hoque, R. Guo \& A. S. \\ Bhalla
}

To cite this article: J. D. S. Guerra, A. C. Silva, R. Mcintosh, Mohammad M. Hoque, R. Guo \& A. S. Bhalla (2015) Investigation of the Physical Properties of PLZT Ferroelectric Ceramics - Effect of the Lanthanum Content, Integrated Ferroelectrics, 166:1, 158-167, DOI: 10.1080/10584587.2015.1092221

To link to this article: https://doi.org/10.1080/10584587.2015.1092221

Submit your article to this journal ${ }^{\pi}$

Џ Article views: 52

View Crossmark data ¿

Citing articles: 3 View citing articles ๘ 


\title{
Investigation of the Physical Properties of PLZT Ferroelectric Ceramics - Effect of the Lanthanum Content
}

\author{
J. D. S. GUERRA, ${ }^{2, *}$ A. C. SILVA, ${ }^{2,3}$ R. MCINTOSH, ${ }^{1}$ \\ MOHAMMAD M. HOQUE, ${ }^{1}$ R. GUO, ${ }^{1}$ AND A. S. BHALLA ${ }^{1}$
}

${ }^{1}$ Multifunctional Electronic Materials and Devices Research Lab. Department of Electrical and Computer Engineering, College of Engineering, The University of Texas at San Antonio, San Antonio, TX 78249, USA

${ }^{2}$ Grupo de Ferroelétricos e Materiais Multifuncionais, Instituto de Física, Universidade Federal de Uberlândia 38408-100, Uberlândia, Minas Gerais, Brazil

${ }^{3}$ Departamento de Física e Química, UNESP, Campus de Ilha Solteira, 15385-000 Ilha, Solteira, São Paulo, Brazil

\begin{abstract}
In this work, the structural, ferroelectric and dielectric properties, including radio-frequencies and microwave region, has been investigated in $\mathrm{Pb}_{1-x} \mathrm{La}_{x}\left(\mathrm{Zr}_{y} \mathrm{Ti}_{1-y}\right)_{1-(x / 4)} \mathrm{O}_{3}($ PLZT $)$ ferroelectric ceramics, where $\mathrm{x}=0.02,0.04,0.06$, $0.08,0.10,0.12$ and $\mathrm{y}=0.70$. Ceramic samples were obtained from the solid-state reaction sintering method, and the dielectric measurements were performed in a wide frequency and temperature region, below and above the paraelectric-ferroelectric (PE$F E)$ phase transition temperature. The structural properties, investigated at room temperature, from the $x$-ray diffraction (XRD) technique, revealed the evolution from the rhombohedral (R3m symmetry) to orthorhombic (Pmmm symmetry) phases, with the increase of the lanthanum concentration. The ferroelectric as well as the low frequency dielectric properties revealed a change in the PE-FE phase transition characteristics from a normal to a relaxor behavior, with the increase of the lanthanum content. On the other hand, the high-frequency (microwave) dielectric properties studied at room temperature, showed a strong dielectric dispersion on both normal $(\mathrm{x} \leq 0.06)$ and relaxor $(\mathrm{x}>0.06)$ compositions, which indeed revealed to be also strongly dependent on the lanthanum concentration.
\end{abstract}

Keywords PLZT; ferroelectric ceramics; phase transition; dielectric properties

Ferroelectric systems have attracted the attention of the scientific community since the past five decades because of their very interesting physical properties, which make them potential materials for practical applications [1]. For instance, because of their excellent ferroelectric, piezoelectric and pyroelectric characteristics they have been widely used in electronic devices such as capacitors, transducers, thermistors, memories, and others [2-5]. Ferroelectrics are characterized by a reversible spontaneous polarization in the absence of an electric field [2,3], which arises from a non-centrosymmetric arrangement of ions in its

Received in final form June 13, 2015.

*Corresponding authors. E-mail: jose.guerra@utsa.edu, santos@infis.ufu.br 
unit cell that produces an electric dipole moment. Adjacent unit cells tend to polarize in the same direction and form a region called a ferroelectric domain [3]. The dynamics as well as the characteristics of the domain structure in ferroelectrics plays a very important role in these materials to be used for practical applications [4, 5]. Above a critical temperature, named as the Curie temperature $\left(T_{\mathrm{C}}\right)$, these materials show a centrosymmetric structure and, therefore, the spontaneous polarization vanishes. In this state, the material is termed paraelectrics. As the temperature is lowered through the Curie point, a phase transition takes place from the paraelectric (PE) state to the ferroelectric (FE) one.

According to the phase transition as well as the domains structure characteristics, ferroelectrics can be classified as either normal or relaxors [2]. In normal ferroelectrics, a well-defined and non-frequency dependent peak of the dielectric permittivity around $T_{\mathrm{C}}$ can be observed $[2,3]$. On the other hand, relaxors are known to exhibit intriguing and very interesting characteristics $[6,7]$, with a very broad and frequency dispersive dielectric permittivity peak. The origin of these unusual physical properties has been discussed in terms of intrinsic heterogeneity at a nanometer scale, with the presence of polar clusters or polar nano-regions (PNR) [7]. Several models have been proposed in order to explain the relaxor behavior of such systems. One of the most acceptable theoretical model, which try to explain the origin and nature of the PNRs, is the dipole-glass model where a glassy state is produced by random fields and random interactions among polar nanometer-sized regions imbedded in a nonpolar matrix $[8,9]$. These characteristics make relaxors attractive materials for application in electronic and optoelectronic devices [10-12]. However, despite of the many possible explanations, the real nature of the relaxor behavior remains still now unclear.

Furthermore, due to their potentiality for application in tunable microwave devices such as phase shifters and antennas [13,14], relaxors have also attracted considerable attention in both bulk and thin film, in the high frequency region. In this way, very high dielectric permittivity, with strong dependence on the applied electric field, and low dielectric losses $(\tan \delta)$ are important characteristics of these materials, which should be taken into account in the device design. To the best knowledge of the present authors no detailed investigations on the physical properties, including microwave dielectric response, of the PLZT system, which consider a very wide lanthanum concentration range, have been reported in the current literature.

The objective of the present work is to investigate the physical properties of $\mathrm{Pb}_{1-x} \mathrm{La}_{x}\left(\mathrm{Zr}_{0.70} \mathrm{Ti}_{0.30}\right)_{(1-x / 4)} \mathrm{O}_{3}$ (PLZT) ceramics, obtained from the conventional solid-state reaction sintering method. The influence of the lanthanum content, with $x=0.02,0.04$, $0.06,0.08,0.10$ and 0.12 , on the structural, ferroelectric and dielectric properties have been taken into account. Specially, the microwave dielectric properties have been investigated in a wide frequency range from $1 \mathrm{MHz}$ up to $1.8 \mathrm{GHz}$.

PLZT ceramics samples, with nominal composition $\mathrm{Pb}_{1-x} \mathrm{La}_{x}\left(\mathrm{Zr}_{0.70} \mathrm{Ti}_{0.30}\right)_{(1-x / 4)} \mathrm{O}_{3}$, for $x=2,4,6,8,10$ and $12 \mathrm{~mol} \%$ La (hereafter labeled as PLZT2, PLZT4, PLZT6, PLZT8, PLZT10 and PLZT12, respectively), were prepared by the conventional solid state reaction method. The corresponding high purity precursor oxides were ball-milled for eight hours, pressed and calcined at $900{ }^{\circ} \mathrm{C}$ for two hours. The calcined powders were milled again for two hours, cold pressed and sintered at $1250{ }^{\circ} \mathrm{C}$ for two hours in $\mathrm{PbO}$ rich atmosphere to prevent $\mathrm{Pb}$ losses. The resulting bulks were cut in pellets of about $1 \mathrm{~mm}$ of thickness. Silver electrodes were applied on both opposite surfaces of the ceramic specimens and then dried at $590^{\circ} \mathrm{C}$. Structural analysis was performed by $\mathrm{x}$-ray powder diffraction (XRD) technique at room temperature, using a SHIMADZU, model XDR-6000, diffractometer with $\mathrm{CuK}_{\alpha 1}\left(1.5406 \AA\right.$ ) radiation in a $2 \theta$ range from $10^{\circ}$ to $80^{\circ}$, with step scan of $0.02^{\circ}$. 


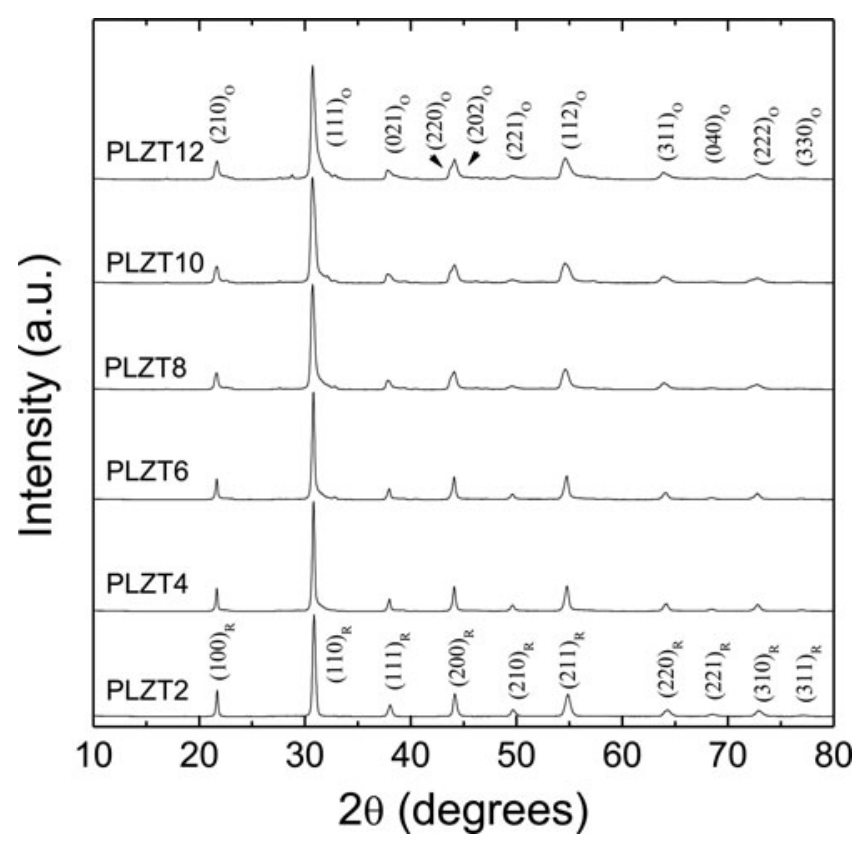

Figure 1. X-ray diffraction (XRD) patterns obtained at room temperature for the studied PLZT compositions.

The relative density of the samples was obtained from the Archimedes' method, using a Mettler Toledo, model AG285, analytical balance. Ferroelectric characterization $(P-E$ curves) was performed at room temperature by using a conventional Sawyer-Tower circuit and a Trek PM04014 High Voltage AC/DC Generator. Dielectric measurements were carried out in a wide frequency and temperature ranges $\left(100 \mathrm{~Hz}-1 \mathrm{MHz}\right.$ and $25-350{ }^{\circ} \mathrm{C}$, respectively) by using a HIOKI 3532-50 LCR HiTESTER meter. On the other hand, the high frequency (microwave) dielectric measurements were collected by using a HP4291A RF Impedance/Material Analyzer, covering a frequency range of $1 \mathrm{MHz}-1.8 \mathrm{GHz}$.

Figure 1 shows the X-ray diffraction measurements obtained for the PLZT powdered ceramic samples. Results confirmed the rhombohedral $(R 3 m)$ ferroelectric phases (represented by indexed peaks), with perovskite structure for the samples with PLZT2 up to PLZT10 compositions, whereas for the PLZT12 sample initial observations suggest also the presence of orthorhombic ferroelectric phase. In this case, detailed studies are needed to confirm this issue. For all the cases, no secondary phases were observed. Detailed information on the structural characteristics were investigated after structural refinement of the experimental data from Reitveld refinement method analyses, carried out by using the GSAS-EXPGUI software [15]. Table 1 shows the lattice parameters, the volume of the unit cell and the Rietveld parameter, obtained for all the samples after structural refinement of the experimental data.

Results revealed structural changes in the PLZT ceramics with the increase of the lanthanum ion. In fact, it was observed that the volume of the unit cell decreases with the increase of the lanthanum content, which can be explained on the basis of the ionic radius. Since in perovskite-type structures $\left(\mathrm{ABO}_{3}\right)$, the lanthanum cation $\left(\mathrm{La}^{3+}\right)$ has ionic radius around $1.36 \AA$ [16], it can easily occupy the A-site, substituting the lead ion $\left(\mathrm{Pb}^{2+}\right)$ with 


\section{Table 1}

Relative density values $(\rho)$, lattice parameters and volume of the unit cell, obtained for all the samples after structural refinement of the experimental data from Rietveld refinement method analyses using the GSAS-EXPGUI software [15]

\begin{tabular}{lccccc}
\hline Composition & Structure & Lattice parameters $(\AA)$ & Volume $\left(\AA^{3}\right)$ & $\mathrm{R}_{\mathrm{wp}}(\%)$ & $\rho(\%)$ \\
\hline PLZT2 & Rhombohedral & $a=b=c=4.1078$ & 69.3 & 5.33 & 90.7 \\
PLZT4 & Rhombohedral & $a=b=c=4.1049$ & 69.2 & 7.75 & 94.3 \\
PLZT6 & Rhombohedral & $a=b=c=4.0873$ & 68.3 & 5.70 & 99.8 \\
PLZT8 & Rhombohedral & $a=b=c=4.0834$ & 68.1 & 7.57 & 96.7 \\
PLZT10 & Rhombohedral & $a=b=c=4.0810$ & 68.0 & 8.67 & 97.9 \\
PLZT12 & Orthorhombic/ & $a=4.0982 ; b=$ & 67.5 & 8.03 & 94.7 \\
& Rhombohedral & $4.0703 ; c=4.0463$ & & & \\
\hline
\end{tabular}

relative similar ionic radius of $1.49 \AA$ [16]. Therefore, lanthanum ions with smaller radius can easily enter into the crystal lattice of the as-prepared PLZT structure-type samples and locate itself in the A-site with adequate space. However, because of the difference in the ionic radius, the replacement of $\mathrm{Pb}^{2+}$ ions in $\mathrm{A}$-sites by $\mathrm{La}^{3+}$ ions will cause the contraction of the unit cell, resulting in the reduction of the lattice parameters and, consequently, a decrease in the unit-cell volume. On the other hand, the obtained relative density values revealed to be higher than $90 \%$ for all the cases, as shown in Table 1.

Figure 2 shows the hysteresis loops obtained for the studied PLZT ceramics at room temperature. As can be observed, the shape of the obtained hysteresis loops seems to change

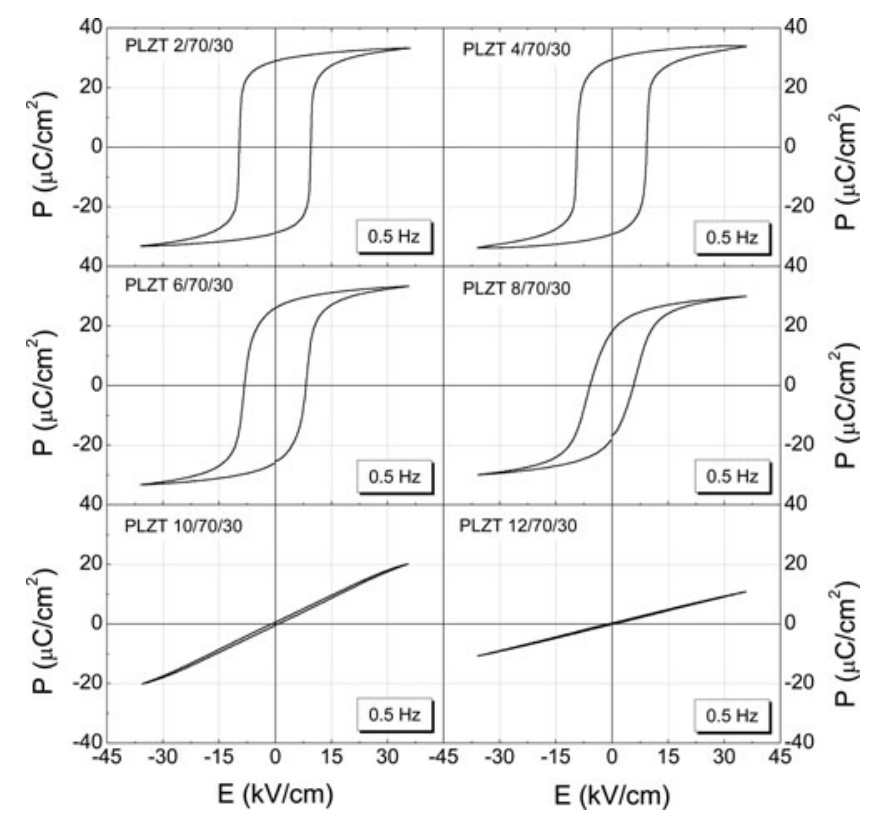

Figure 2. Ferroelectric hysteresis loops $(P-E)$ obtained at room temperature, for the studied PLZT compositions. 
from a square-like to slim loop with the increase of the lanthanum composition. At the same time, it can be noticed that the remnant polarization $\left(\mathrm{P}_{\mathrm{R}}\right)$, as well as the coercive field $\left(\mathrm{E}_{\mathrm{C}}\right)$, decreases with the increase of the lanthanum content.

Indeed, the obtained result suggests a gradual transition from a normal ferroelectric, for the PLZT2 composition, to a relaxor-like characteristics system, for the PLZT12 sample. It has been previously reported, from elastic diffuse neutron and $\mathrm{x}$-ray scattering studies $[17,18]$, that for some lanthanum concentrations the distortion of the crystalline lattice in the PZT system due to the ion displacement could promote the formation of the so-called polar nano-regions (PNRs); the basic ideas about the nature of the relaxor behavior have been related to the dynamics and formation of the PNRs.

Figure 3 shows the temperature dependence of the real $\left(\varepsilon^{\prime}\right)$ and imaginary $\left(\varepsilon^{\prime \prime}\right)$ components of the dielectric permittivity, measured at different frequencies, for the PLZT2 and PLZT10 samples, as example of the obtained results for all the studied compositions. As can be observed in Fig. 3(a), for the lower lanthanum composition sample (PLZT2), the dielectric response shows typical characteristics of a normal paraelectric-ferroelectric (PEFE) phase transition, where the temperature of the maximum real dielectric permittivity $\left(\mathrm{T}_{\mathrm{m}}\right)$ corresponds to the PE-FE phase transition temperature, known as Curie temperature $\left(T_{C}\right)$. In these cases, the temperature of the maximum dielectric permittivity does not show any frequency dependence, whereas the temperature of the maximum imaginary dielectric

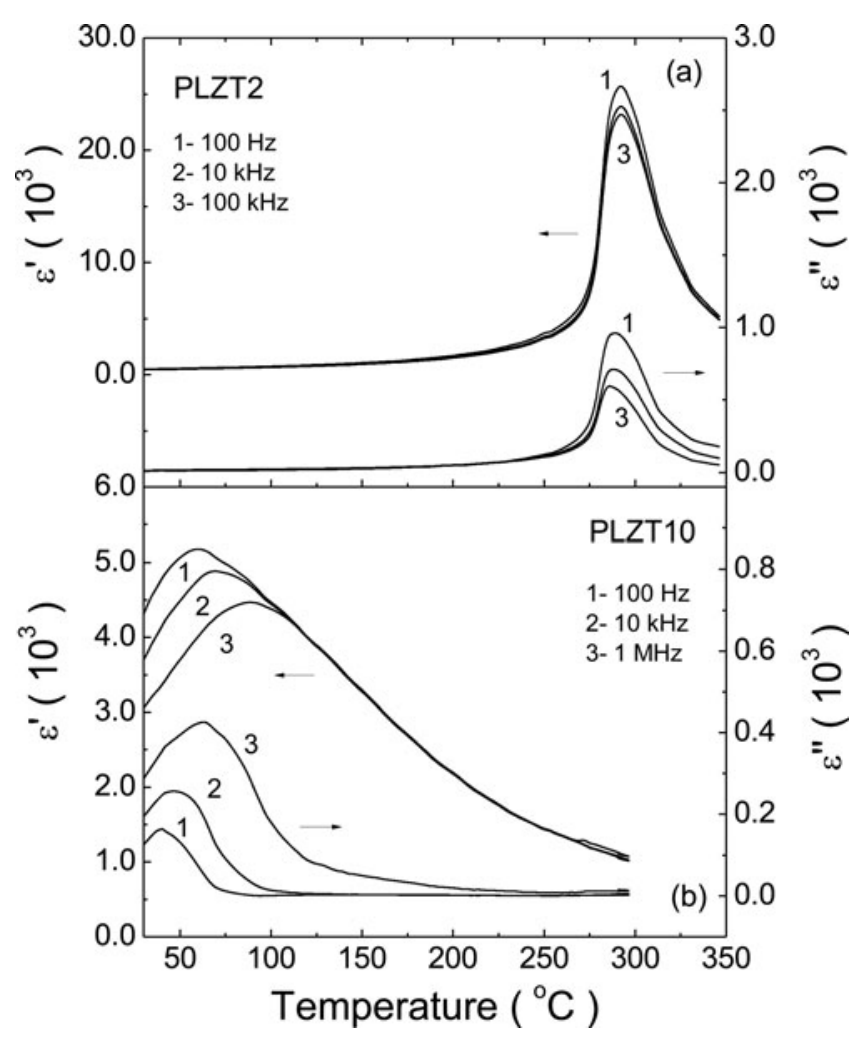

Figure 3. Temperature dependence of the real $\left(\varepsilon^{\prime}\right)$ and imaginary $\left(\varepsilon^{\prime \prime}\right)$ component of the dielectric permittivity, for the PLZT2 and PLZT10 samples, at several frequencies, as example of the obtained results for all the studied compositions. 
Table 2

Dielectric parameters (maximum dielectric permittivity and its corresponding temperature: $\varepsilon_{\mathrm{m}}$ and $\mathrm{T}_{\mathrm{m}}$, respectively) for the studied PLZT compositions, obtained at $100 \mathrm{~Hz}$

\begin{tabular}{lcr}
\hline Composition & $\mathrm{T}_{\mathrm{m}}\left({ }^{\circ} \mathrm{C}\right)$ & $\varepsilon_{\mathrm{m}}$ \\
\hline PLZT2 & 292 & 25750 \\
PLZT4 & 236 & 16224 \\
PLZT6 & 172 & 12551 \\
PLZT8 & 101 & 9252 \\
PLZT10 & 60 & 5184 \\
PLZT12 & 31 & 2445 \\
\hline
\end{tabular}

permittivity coincides with $\mathrm{T}_{\mathrm{m}}$. However, similar to other classical relaxor ferroelectrics [8], for higher lanthanum concentrations (PLZT6, PLZT8, PLZT10 and PLZT12) the dielectric response, which has been here depicted in Fig. 3(b) for the PLZT10 composition, exhibits a pronounced frequency dispersion in the complex dielectric permittivity with a very broad peak around the temperature of the maximum real dielectric permittivity. As observed in Fig. 3(b), the maximum real dielectric permittivity decreases, while the maximum imaginary dielectric permittivity increases with the increase of the frequency, and the temperature of the maximum dielectric permittivity, in both cases (real and imaginary components), increases with increasing frequency. On the other hand, it can also be observed that the maximum of the imaginary component of the dielectric permittivity is reached at a temperature lower than that obtained for the maximum real dielectric permittivity. As aforementioned, these diffuse phase transition behaviors are the characteristics of relaxor ferroelectrics $[6,7]$. It is worth to point out that signature of relaxor characteristics have been observed for the PLZT12 composition. However, both the maximum for $\varepsilon^{\text {' }}$ and $\varepsilon^{\text {" }}$ components were observed near the room temperature, which is close to the lower limit of the measured temperature range allowed by our experimental system.

The origin of the relaxor behavior has been investigated over the last three decades and several models have been proposed. Relaxor systems do not undergo a macroscopic phase transition from the paraelectric phase into a ferroelectric state one, but they become rather "frustrated" ferroelectrics. They are complex materials where the origin of the "frustration" has been associated with compositional inhomogeneity on a nanometer scale that results from partial compositional disorder in a specific lattice site. This disorder prevents the macroscopic transformation into ferroelectric phase. A picture coherent with the experimental results is the presence of a paraelectric matrix, in which ferroelectric nanosized regions are formed upon cooling, without the evolution of a coherent macroscopic ferroelectric phase.

On the other hand, it can also be noticed from Fig. 3 that the broadness of the peak around $\mathrm{T}_{\mathrm{m}}$ increases with the increase of the lanthanum content, revealing a broadened response for the higher lanthanum concentrations. The random occupation of some equivalent lattice sites by different ions (compositional disorder) has been ascribed as the main cause of the observed behavior [6, 7]. Contrary to normal ferroelectrics, where the PE-FE phase transition is characterized by a sharp peak of the dielectric permittivity, relaxors exhibit a very broad and frequency dependent maximum dielectric permittivity, which is known as diffuse phase transition (DPT). Most of the proposed models for relaxors postulate the 


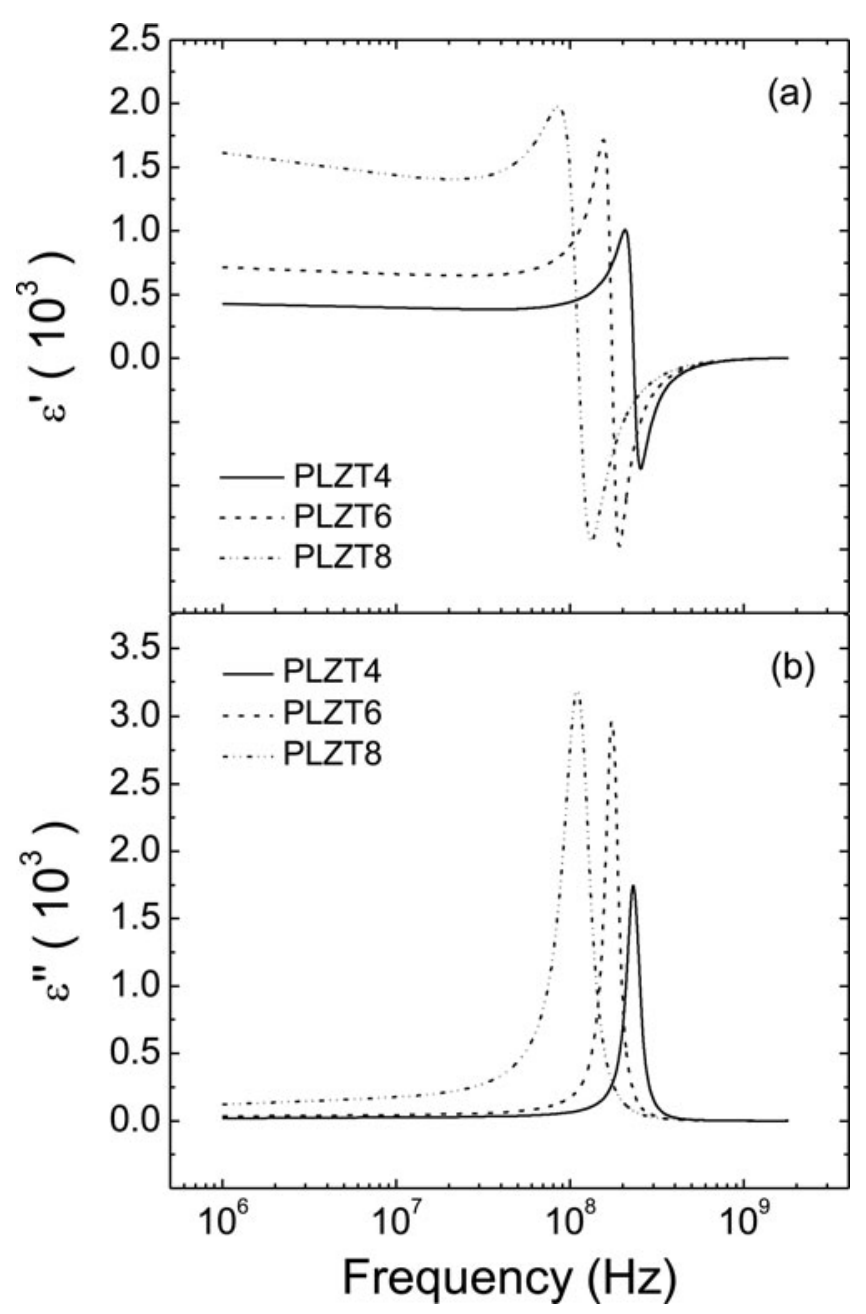

Figure 4. Frequency dependence of the real $\left(\varepsilon^{\prime}\right)$ and imaginary $\left(\varepsilon^{\prime \prime}\right)$ component of the dielectric permittivity, for some selected PLZT compositions, at room temperature.

formation of randomly oriented order-disorder PNRs in sizes as the origin of the DPT. The polar regions are considered as the nuclei of ferroelectric phase in a paraelectric matrix and since the ferroelectric transition temperature $\left(T_{m}\right)$ is strongly dependent on the composition and sizes, the different PNRs will have different frequency response and $T_{m}$ values. As a results, a broader peak of the dielectric permittivity around $T_{m}$ for different frequency is observed.

Table 2 shows the dielectric parameters obtained from the experimental results of the dielectric response for all the studied compositions. As can be seen, the temperature of the maximum dielectric permittivity $\left(\mathrm{T}_{\mathrm{m}}\right)$ decreases with the increase of the lanthanum content, showing a linear-like dependence, which confirms the high solubility of the lanthanum cation in the crystalline lattice of the PLZT system.

Figure 4 depicts the frequency dependence of the real and imaginary components of the dielectric permittivity, at high frequencies (microwave) region, for some selected 


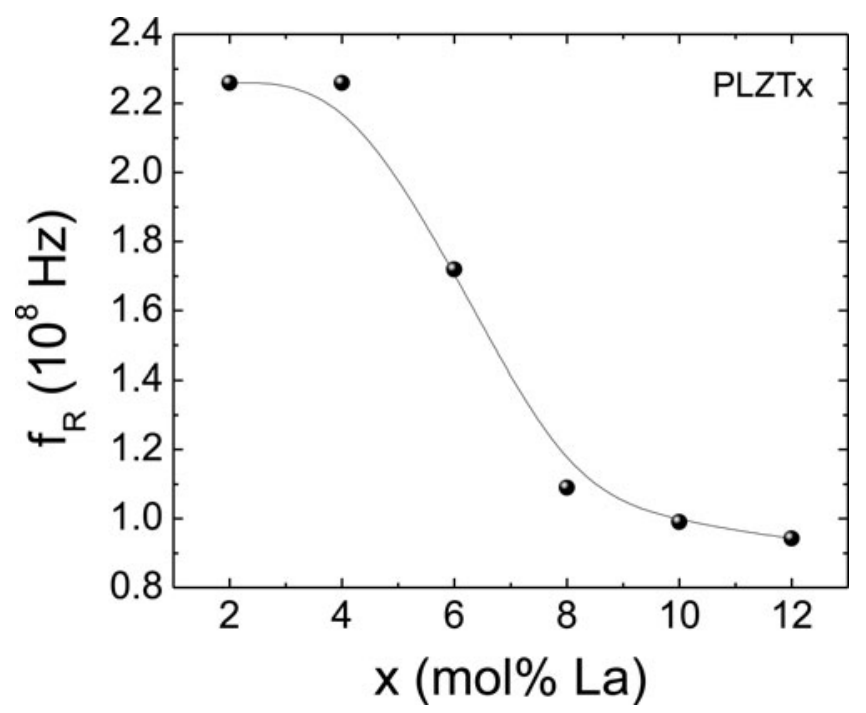

Figure 5. Dependence of the characteristic frequency $\left(f_{R}\right)$ values obtained from the experimental data of Fig. 4, for all the studied compositions.

compositions (PLZT4, PLZT6 and PLZT8). As can be observed, strong dielectric anomalies with typical resonance behaviors were obtained for all the investigated samples. Taking as example the result obtained for the PLZT4 sample, it can be seen that the real component of the dielectric permittivity $\left(\varepsilon^{\prime}\right)$ remains almost constant over a wide frequency range, then starts to increase for frequencies higher than $0.8 \times 10^{9} \mathrm{~Hz}$, traversing a maximum and then decreases up to its clamped value. In this process, the imaginary dielectric permittivity $\left(\varepsilon^{\prime \prime}\right)$ shows its maximum value at around $2.25 \times 10^{8} \mathrm{~Hz}$ and continuously decreases. The frequency corresponding to the maximum imaginary dielectric permittivity is known as the characteristic frequency $\left(f_{\mathrm{R}}\right.$, where $\left.\omega_{\mathrm{R}}=2 \pi f_{\mathrm{R}}\right)$ and it is related with the polarization mechanism responsible for the dissipation at high frequencies.

The observed behavior, which has been mainly attributed to several factors such as piezoelectric resonance of grains [21] and/or individual domains [22], inertial component of domain walls [23], and to the correlated hopping of off-centered ferroelectric active ions between several potential wells [24], seems to be a characteristic response for typical ferroelectric systems $[19,20]$, being this anomaly directly linked to the domain state of the ferroelectric phase. In this context, a more general description recently reported by Guerra et al. considers the vibration of the boundaries of polar regions being the common mechanism responsible for the microwave dielectric dispersion processes in perovskite ferroelectric systems [25, 26], where the observed anomalies are governed by an overdamped resonance mechanism associated with the polar regions [26].

Figure 5 shows the experimental values of the characteristic frequency $\left(f_{R}\right)$ for all the studied compositions, obtained from the maximum peak for the imaginary dielectric permittivity of Fig. 4. Result shows a strong variation of the characteristic frequency with the increase of the lanthanum content, revealing that the inclusion of the lanthanum cations in the PZT system has a noticeable contribution on the high-frequency dielectric response of the studied PLZT system, directly affecting the ferroelectric domain-wall motion dynamics of the ferroelectric PLZT phase. 
From the application point of view, the study of the high frequency dielectric parameters plays a very important role, in order to contribute for the understanding of the real nature of the observed anomalies and distinguishing different observed behaviors at microwave frequency in these materials. Distinctions between the contributions of relaxation-like and/or resonance responses are very important for materials development, specially, in relaxor systems, where their physical properties are strongly dependent on the structural characteristics. Further studies are in progress in order to closely treat these aspects, taking into account the fitting of the experimental data by using the theoretical model previously reported in the literature $[25,26]$.

In summary, the physical properties of PLZT ferroelectric ceramics, obtained from the conventional sintering method, have been investigated taking into account the lanthanum concentration. Structural analysis revealed a contraction in the volume of the unit-cell as the lanthanum content increases, which can be explained on the basis of the ionic radii. The ferroelectric properties, as well as the low-frequency dielectric response, confirmed an evolution from normal paraelectric-ferroelectric phase transition to a relaxor behavior, with the increase of the lanthanum concentration, which can be related to the dynamics and formation of the PNRs arisen from the order-disorder or the compositional inhomogeneity. On the other hand, the La substitution into Pb-site promoted the linear-like decrease of the transition temperature, which is a strong evidence of the high solubility of the La in the PLZT crystalline lattice. The high-frequency dielectric measurements revealed strong dielectric dispersion with resonance characteristics, which could be related to an over-damped resonance mechanism for the vibration of the boundaries of polar-regions, characteristics of the ferroelectric phase. The strong dependence of the characteristic frequency with the lanthanum concentration also confirms the noticeable contribution of the microstructural characteristics on the dynamic of the domain-wall motion. These insights provide additional features for the use of such PLZT compositions in high-frequency application devices.

\section{Funding}

The authors would like to thank CNPq and FAPEMIG Brazilian agencies, and INAMM/NSF (Grant No. 0884081), for the financial support. A. C. Silva also thanks the Materials Science Post-Graduation Program (PPGCM), UNESP, Ilha Solteira, Brazil.

\section{References}

1. K. Uchino, Ferroelectric Devices (New York: Marcel Dekker Inc.) 2000.

2. M. E. Lines, and A.M. Glass, Principles and Applications of Ferroelectric and Related Materials (Oxford: Clarendon Press), 1997.

3. B. Jaffe, W. R. Cook, and H. Jaffe, Piezoelectric Ceramics (London: Academic Press) 1971.

4. J. C. Burfoot, and G. W. Taylor, Polar Dielectrics and Their Applications (Berkeley: Univ. of Calif. Press) 1979.

5. J. M. Herbert, Ceramic Dielectrics and Capacitors (New York: Gordon and Breach) 1985.

6. G. A. Smolenskii, Physical phenomena in ferroelectrics with diffused phase transition. J. Phys. Soc. Jpn. 28, 26-37 (1970).

7. L. E. Cross, Relaxor Ferroelectrics. Ferroelectrics 76, 241-267 (1987).

8. D. Viehland, S. J. Jang, L. E. Cross, and M. Wuttig, Freezing of the polarization fluctuations in lead magnesium niobate relaxors. J. Appl. Phys. 68, 2916-2921 (1990).

9. R. Pirc, and R. Blinc, Spherical random-bond-random-field model of relaxor ferroelectrics. Phys. Rev. B 60, 13470-13478 (1999). 
10. Y. Yokomizo, T. Takahashi, and S. Nomura, Ferroelectric properties of $\mathrm{Pb}\left(\mathrm{Zn}_{1 / 3} \mathrm{Nb}_{2 / 3}\right) \mathrm{O}_{3}$. J. Phys. Soc. Jpn. 28, 1278-1284 (1970).

11. V. Westphal, W. Kleemann, and M. D. Glinchuk, Diffuse phase transitions and random-fieldinduced domain states of the "relaxor" ferroelectric $\mathrm{PbMg}_{1 / 3} \mathrm{Nb}_{2 / 3} \mathrm{O}_{3}$. Phys. Rev. Lett. 68, 847-850 (1992).

12. A. E. Glazounov, and A. K. Tagantsev, A "breathing" model for the polarization response of relaxer ferroelectrics. Ferroelectrics 221, 57-66 (1999).

13. Tanmoy Maiti, R. Guo, and A. S. Bhalla, Electric field dependent dielectric properties and high tunability of $\mathrm{BaZr}_{x} \mathrm{Ti}_{1-x} \mathrm{O}_{3}$ relaxor ferroelectrics. Appl. Phys. Lett. 89, 122909 (2006).

14. Tanmoy Maiti, R. Guo, and A. S. Bhalla, Enhanced electric field tunable dielectric properties of $\mathrm{BaZr}_{x} \mathrm{Ti}_{1-x} \mathrm{O}_{3}$ relaxor ferroelectrics. Appl. Phys. Lett. 90, 182901 (2007).

15. A. C. Larson, and R. B. Von Dreele, General Structure Analysis System (GSAS), Los Alamos National Laboratory Report LAUR 86-748 (2004).

16. K. Ramam, and K. Chandramouli, Dielectric and piezoelectric properties of combinatory effect of a-site isovalent and b-site acceptor doped PLZT ceramics. Ceramics-Silikáty 53, 189-194 (2009).

17. A. A. Bokov, Recent advances in diffuse ferroelectric phase transitions. Ferroelectrics 131, 49-55 (1992).

18. V. Bovtun, J. Petzelt, V. Porokhonskyy, S. Kamba, and Y. Yakimenko, Structure of the dielectric spectrum of relaxor ferroelectrics. J. Eur. Ceram. Soc. 21, 1307-1311 (2001).

19. J. D. S. Guerra and J. A. Eiras, Dielectric anomalies in La modified $\mathrm{PbTiO}_{3}$ ferroelectric ceramics in the microwave frequency region. Ferroelectrics 294, 25-31 (2003).

20. S. Tappe, U. Böttger, and R. Waser, Electrostrictive resonances in $\left(\mathrm{Ba}_{0.7} \mathrm{Sr}_{0.3}\right) \mathrm{TiO}_{3}$ thin films at microwave frequencies. Appl. Phys. Lett. 85, 624-626 (2004).

21. A. V. Hippel, Piezoelectricity, ferroelectricity, and crystal structure. Z. Phys. 133, 158-173 (1952).

22. C. Kittel, Domain boundary motion in ferroelectric crystals and the dielectric constant at high frequency. Phys. Rev. 83, 458 (1951).

23. A. V. Turik, and N. B. Shevchenko, Dielectric spectrum of $\mathrm{BaTiO}_{3}$ single crystals. Phys. Stat. Sol. B 95, 585-592 (1979).

24. L. Zhang, W. L. Zhong, C. L. Wang, P. L. Zhang, and Y. G. Wang, Dielectric relaxation in barium strontium titanate. Solid State Commun. 107, 769-773 (1998).

25. J. D. S. Guerra, M. H. Lente, and J. A. Eiras, Microwave dielectric dispersion process in perovskite ferroelectric systems. Appl. Phys. Lett. 88, 102905 (2006).

26. J. D. S. Guerra and J. A. Eiras, Mechanical and electrical driving field induced high-frequency dielectric anomalies in ferroelectric systems. J. Phys.: Condens. Matter 19, 386217 (2007). 